\title{
Abstract
}

Fitness apps are promising digital tools to support self-tracking and physical activity. Specific app functions such as normalized step targets represent controlling conditions that can affect controlled vs. autonomous motivation and thus motivated physical activity. It was the aim of this study to examine the effects of self-tracking via a fitness app and the implementation of a normalized step target on aspects of motivation and physical activity by using Self-Determination Theory as a theoretical framework. In a six-week RCT $(N=152$; age $M=24.43, S D=4.61)$, participants in two groups were provided with fitness app devices to track their physical activity. Participants in one group (ET) had a normalized step target of 10,000 whereas the other group (ENT) had not any step target. Participants in a third control group tracked their physical activity without fitness app support. Self-reported physical activity, basic needs satisfaction, and motivation regulation were assessed.

Physical activity increased in the fitness tracker groups compared to the control group. Moderate physical activity and autonomy need satisfaction increased in the ENT group. Identified motivation decreased in the ET and control groups and introjected motivation decreased in the control group. Amotivation increased in the ENT group. Conclusively, self-tracking via fitness apps can support physical activity, and normalized step targets can undermine motivation. Lack of normalized targets can support autonomy need satisfaction and physical activity but can also foster amotivation. Thus, it is advised to support autonomous goal setting in fitness app users.

Keywords: motivation, fitness app, physical activity, Self-Determination Theory, autonomy 


\section{Normalised Step Targets in Fitness Apps Affect Users' Autonomy Need Satisfaction, Motivation and Physical Activity - A Six-Week RCT}

\section{Introduction}

Over the past decades, overweight and obesity have increased and are highly prevalent across developed western societies (WHO, 2017). Based on recent data, $68 \%$ of adults in the United States and $57 \%$ of adults in Germany are overweight, and about $13 \%$ of the world's adult population have been found to be obese in 2016 (WHO, 2017). Overweight has been associated with an increased risk of multiple diseases, including type-II diabetes, heart diseases, and high cholesterol, posing comprehensive epidemiologic problems on individual and political levels (Field et al., 2001; Prugger \& Keil, 2007; WHO, 2017). Also, prevalence of overweight and obesity have been associated with lower socioeconomic status (Helmert \& Strube, 2004), raising the need to implement low-cost and low-threshold options targeting overweight in the broad society. With the aim to reduce epidemiologic problems associated with overweight and obesity, it has been recommended that people engage in moderate exercise of about 150 minutes per week in units of at least ten minutes, for example five times a week for 30 minutes (Warburton, Charlesworth, Ivey, Nettlefold, \& Bredin, 2010) or 75 minutes of vigorous intensity (Bouchard et al., 2018). Alternatively, coverage of 10,000 steps per day have been recommended (Bouchard et al., 2018; Tudor-Locke et al., 2010). In this context, the ongoing and dynamic increase in digitalization creates the opportunity for large frequency smartphone-based interaction and new options for health behaviour interventions (Statista, 2018; West et al., 2012). Thus, health practitioners have become interested in implementing health behaviour related options and gadgets (Riley, Lee, Cooper, Fairburn, \& Shafran, 2007; West et al., 2012). The prominent formation of such implementations has led to the establishment of electronic health (e-health) and mobile health (m-health). Here, fitness 
apps are the most popular and widely used apps in the health app sector. For example, normalized step targets (e.g., 10,000 steps per day) have been implemented in fitness apps to support health behaviour.

\section{Fitness apps}

Fitness apps are specific applications that can be used on a smartphone to track health related parameters in daily life, such as step coverage, calorie consumption, nutrition, etc. (Poushter, 2016; West et al., 2012). With the aim to enhance physical activity in digital age, fitness apps represent a low-cost and easily applicable opportunity to provide support in initiation and maintenance of physical activity (West et al., 2012; Schoeppe et al., 2016). In an international comparison across developed countries, $33 \%$ of the participants stated to currently track their health or fitness via an app, fitness band or smartwatch (Global Studies-Fitness tracking, 2017). However, high dropout rates in fitness app usage have been observed, i.e., an average of $18 \%$. As reviewed by Schoeppe et al. (2016), a decline in fitness app usage occurs during intervention studies after five to six weeks. Thus, it is of high interest to investigate the effects of specific healthy technology and options (i.e., fitness app usage) on health behaviour and also to understand the determinants of motivated and maintained fitness app usage.

In sport and exercise psychology, the effects of fitness app usage have been subject to a growing body of studies (Romeo, 2019; Schoeppe et al., 2016). It has been shown that fitness app usage can promote higher activity levels, such as step counts (Glynn et al., 2014; Goodyear et al., 2017; Stawarz, Cox, \& Blandford, 2015). Reviewing the efficacy of fitness app based interventions, Schoeppe et al. (2016) found that improvements in physical activity were observed in 14 out of 21 studies. In a meta-analysis targeting RCT studies with objective measures of physical activity, it was found that the step count did not increase in fitness app users compared to controls. Furthermore, it was found that short-term interventions of less than three months were more effective than long-term interventions, and that apps targeting 
physical activity were more effective than those directing at a combination of diet and physical activity (Romeo, 2019). Also, the effects did not differ with regards to the target population. However, only six studies could be included in the analysis as high quality studies using RCT designs and applying objective measures are still lacking.

Fitness apps have been designed and are used to change health behaviour, for example to be more active or to lose weight (Busch, 2019; Higgins, 2016; West et al., 2012). Thus, most fitness app interfaces include options that target one or multiple points of behaviour change. Most frequently, aspects of self-monitoring, goal setting, and performance feedback are implemented (Middelweerd et al., 2014). In this context, has been systematically reviewed that interventions using such behaviour change techniques were more effective compared to interventions using no such techniques (Schoeppe, 2016). In the context of specific behaviour change theories, researchers have also focused on motivational factors predicting exercise behaviour in fitness app users. For example, high levels of self-determined motivation have been associated with exercise behaviour in fitness tracker users (Donnachie et al., 2017; Kerner \& Goodyear, 2017) beyond the background of the Self-Determination Theory (SDT; (Deci \& Ryan, 1985; R. Ryan \& Deci, 2017). SDT is a comprehensive and widely used theoretical framework that has also been adapted to the exercise context (SDT Health Model; Ryan et al., 2008) and provides a valuable framework to investigate motivational processes in connection with maintenance of fitness app usage.

\section{Self-Determination Theory}

SDT (Deci \& Ryan, 1985; R. Ryan \& Deci, 2017) is a macro-theory that has been widely used to describe and explain motivational processes predicting motivated behaviour in multiple contexts, such as work, education, clinical psychology, and sport and exercise psychology (Gagné \& Deci, 2005; Niemiec \& Ryan, 2009; Ntoumanis, 2001; Ryan \& Deci, 2008). On the particular field of motivated health behaviour, SDT has been adapted on 
theoretical level (Ryan et al., 2008) and has been applied on empirical level to gain valuable knowledge about the practice and maintenance of self-determined physical activity, sedentary behaviour, and mental health behaviour. Fundamentally, the SDT health model incorporates the prediction of health behaviour via a continuum of exercise regulation modes and exercise specific basic needs satisfaction that can be influenced by environments and other factors that support the basic need satisfaction in an individual.

\section{Basic need satisfaction}

According to SDT, self-determined motivation is most likely when three human basic needs are satisfied, i.e., autonomy, competence, and relatedness needs. Autonomy need satisfaction reflects the experience of own choice about behaviour and can be positively influenced by environments and conditions that are autonomy supportive for an individual. Competence need can be satisfied by experiencing competence about one's behaviour, and relatedness need satisfaction reflects the experience of connection to other people. The higher the basic needs can be satisfied in a specific context, the more the related behaviour can be experienced as joyful, and thus is likely to lead to autonomous forms of motivation regulation and to motivated behaviour.

\section{Motivation regulation}

Satisfaction of basic needs is likely to lead to high levels of autonomous forms of motivation (i.e., intrinsic and identified motivation regulation) and to low levels of controlled regulation (i.e., introjected and external motivation) or amotivation (Ryan \& Deci, 2017; Teixeira et al., 2012). Motivation can be described on a continuum of regulation modes relating the concordance of behaviour to personal values and interests. First, intrinsic motivation is the most self-determined form of motivation. Intrinsic motivation is characterized by high inherence with personal goals and is experienced as joyful. Second, identified motivation is characterized as congruent with goals that are believed to be important (e.g., valuing the 
benefits of exercise). Third, introjected regulation reflects a low inherence with personal goals, but is regarded as important (e.g., feeling guilty when not exercising). Fourth, external motivation is not congruent with personal goals and is based on compliance to external controls (e.g., pressure, fear of negative consequences when not exercising). Beyond motivated regulation, amotivation is described as a state of lacking intention to act (e.g., not seeing the point in exercising). Current research has provided extensive support of the SDT health model to explain motivational processes leading to the initiation and maintenance of physical activity (Ng et al., 2012; Teixeira et al., 2012). Specifically, exercise basic needs satisfaction and self-determined motivation have been shown to be influential predictors of initiation and maintenance of health behaviour ( $\mathrm{Ng}$ et al., 2012; Ryan et al., 2008). Also, the engagement in autonomously motivated exercise compared to controlled motivated exercise has been found to be associated with exercise adherence and that this effect is mediated by positive affect (Silva et al., 2010; Williams et al., 2016).

\section{SDT and fitness app usage}

Examining SDT in digital media and exercise context, intervention studies have investigated the effects of healthy technology usage (i.e., fitness apps) on motivation and physical activity. For example, it has been found that self-tracking of steps via pedometers with autonomous goal-setting led to increased physical activity and facilitated the internalization of motivation during a weight loss program (Donnachie et al., 2017). Similarly, in an intervention with 1314-year olds using Fitbits that had a pre-defined step-target implemented, increases in physical activity were found. However, basic needs satisfaction and autonomous motivation decreased after six weeks, and controlled motivation and amotivation increased (Kerner \& Goodyear, 2017). The authors assumed that basic needs satisfaction had been undermined by the controlled normalized goal setting incorporated in the fitness app. In another study investigating the effects of fitness app usage, Kerner, Burrows, and McGrane (2019) found- 
however insignificant — decreases in autonomous motivation and increases in controlled motivation and amotivation after five weeks' Fitbit usage. After the intervention time, significant decreases in physical activity were observed that were discussed to result from the changes in motivation during fitness app usage. Thus, across studies, mixed effects of fitness tracker usage and specific app functions (e.g., the implementation of a normalized step target) on physical activity, basic needs satisfaction, and motivation in fitness app users were found. Nonetheless, the results indicate that variations in conditions and specific app functions (i.e., an implementation of step targets providing controlled vs. autonomous conditions) can be of high relevance when explaining basic needs satisfaction, autonomous vs. controlled forms of motivation, leading to enhanced or decreased physical activity. Thus, high quality studies using experimental designs such as randomly controlled trials (RCT) that systematically vary specific (e.g., autonomy supportive vs. controlling) app functions (e.g. step targets) that are based on motivation theory foundation are required. Explaining the increased activity levels in fitness app users found in most studies, the practice of self-monitoring has been identified as a behaviour change mechanism (Middelweerd et al., 2014). Although there is a consensus that most fitness app interventions can lead to increased levels of physical activity (Maher et al., 2015; Romeo et al., 2019), there is a lack of studies targeting the effect of the technology specific effects of self-tracking via healthy technology such as fitness apps compared to physical activity tracking without using such healthy technologies. Thus, to understand potential effects, it is required to conduct controlled studies with an additional control group with participants who track their physical activity without technology support.

\section{Objective}

Fitness apps can be promising tools to implement healthy behaviour in the broad population (Middelweerd et al., 2014; Schoeppe et al., 2016; West et al., 2012). However, it has been indicated that the systematic examination of specific app functions (e.g., goal setting via 
normalized step targets) can be of high relevance to understand diverse motivational processes in fitness app users leading to motivated and maintained physical activity vs. amotivation and even decreases in physical activity (e.g., Kerner et al., 2019). Consequently, it is important to implement studies (1) with a theoretical foundation of behaviour change models explaining maintained and motivated exercise behaviour; (2) with randomized controlled conditions; (3) contrasting specific app functions such as normalized step targets vs. no such implementation; (4) with groups practicing self-monitoring via a fitness app vs. no technology mediated support to understand the technology-mediated effect on behaviour change. Therefore, it was the aim of this RCT to investigate the specific effects of selftracking via fitness apps compared to self-tracking without fitness apps and the implementation of a specific normalized target (i.e., 10,000 steps per day) on basic needs satisfaction, motivation, and physical activity using the well-established SDT as a theoretical framework. Beyond the background of the theoretical basis of SDT and the results of studies investigating the effects of fitness app usage using SDT as a framework theory, it was hypothesized:

Hypothesis 1: The implementation of a fitness app tracking device leads to an increased physical activity compared to a control group practicing self-tracking without a selftracking device.

Hypothesis 2: The implementation of a controlled step target in the fitness app leads to a decrease in autonomy need satisfaction compared to no such target.

Hypothesis 3: The implementation of a controlled step target in the fitness app leads to a decrease in autonomous forms of motivation compared to no such target.

Hypothesis 4: The implementation of a controlled step target in the fitness app leads to an increase in controlled forms of motivation and amotivation compared to no such target. 


\section{Materials and Methods}

\section{Trial design}

This study was an intervention study with two randomized experimental groups and one control group. The participants in both experimental groups used a wearable fitness app device to track their physical activity. In one group (Experimental Target group; ET group) a normalized step target of 10,000 steps per day was implemented in the fitness app. In the second group (Experimental No Target group; ENT group), no step goal was set in the app and the participants were asked not to set own goals (see Figure 1 for a visualization of the interface). The additional control group did not receive a wearable fitness app device. In all three groups, the participants tracked their physical activity via a daily diary.

The intervention time was determined six weeks (i.e., 40 days). The decision was based on the following points: (1) The intervention time of six weeks is comparable to studies investigating the effects of fitness app usage (Walsh et al., 2016; Wang et al., 2015); (2) it has been reviewed that a substantial increase in dropout from fitness app usage occurs during intervention studies after about five to six weeks' time (Schoeppe et al., 2016). The study was registered at the German Clinical Trials Register (DRKS, 2019; Grant no. DRKS00014835) and can be viewed at the WHO website http://apps.who.int/trialsearch/. All variables assessed in the study can be viewed here. The study was approved by the ethics committee of the University of Münster. Conduct and reporting of the trial was guided by the Consolidated Standards of Reporting Trials (CONSORT; Schulz, Altman, \& Moher, 2010). Part of the data set used for in this study has been used for a study investigating the effects of fitness app usage on psychological well-being and aspects of body awareness (study blinded for review). The outcome variables assessed in this study have not been used in any other study.

\section{Participants and sample size}

Participants were recruited via flyers distributed at the University of (blinded for review), via 
social media, and via local newspapers. Participants allocated to the experimental groups were informed in advance that the study participation would entail the use of a wearable fitness tracker and that this would result in the disclosure of personal data. For incentivization, every $20^{\text {th }}$ participant completing the study won $20 €$. Each participating student enrolled at the University of (blinded for review) had the alternative opportunity to earn a credit of up to 5.5 hours. Due to the differences in incentives, we also controlled for student status by assessing the status and entering it as a covariate in additional analyses.

The required number of participants was estimated on the basis of a review of studies investigating the effect and dropout rate of fitness app usage on physical activity and motivation in a comparable intervention time (Kerner \& Goodyear, 2017; Schoeppe et al., 2015). Accordingly, a medium size effect of $f^{2}=.18$ was anticipated. A power analysis revealed that a sample size of $n=45$ per group was required to detect a statistical effect of $f^{2}$ $=.18$, given $80 \%$ power, and $\alpha=.05$ (Soper, 2012). Based on reviewed research (Schoeppe et al., 2016), the dropout rate was estimated $10 \%$. Thus, according to the pre-registration of this study, it was planned to recruit 50 participants per group. In a preliminary online questionnaire, the inclusion criteria were screened. The pre-defined inclusion criteria were as follows. The participants (1) are between 18 and 40 years old, representing the main age group using fitness apps (Statista, 2017); (2) self-report exercise behaviour of less than four hours per week on average to focus on participants who are not engaging in professional sport; (3) have not used a fitness app for longer than two weeks within the past six months and thus have similar experience with self-tracking devices; (4) have a smartphone with internet connection and Bluetooth function to meet the technical requirements; (5) are currently not injured or diseased; (6) are not planning to travel for more than seven days during the study period; (7) are not engaging in an employment requiring night shifts on a 
regular basis to ensure valid daily measurement in the fitness app. If the participants met the inclusion criteria, they were randomly assigned to the groups.

\section{Group allocation and interventions}

All participants meeting the inclusion criteria in the screening questionnaire were contacted and invited to the lab. Meanwhile, participants were randomly assigned to the intervention groups. The generation of a sequence allocation was conducted on the basis of the participants' codes. The computer-based pre-test-questionnaires were filled in under controlled lab conditions. The questionnaires were provided via the online survey program unipark (Questback GmbH, 2018). All participants signed informed consent and were asked to contact the researchers in case of technical issues or other problems. Participants allocated to the experimental groups received a fitness tracker device (Fitbit Flex 2) which was set up at the lab. Additionally, the participants received instructions about the fitness tracker usage. The participants could monitor their daily coverage of steps, calories, distance, and active minutes via the app interface. In the ET group, a normalized step target of 10,000 steps per day was set, and the progress of reaching the step target was indicated via the progress bar on the interface (see Figure 1). In the ENT group, no daily step target was set. Participants in both groups were asked not to change the app settings including the step target. During the intervention, the participants were asked to wear the tracking devices all day long. After the six-week intervention, all participants were invited to the lab to fill in the computer-based post-test questionnaire and to return their device. The questionnaire entailed a check, assessing whether the participants had worn the fitness trackers and whether they had changed the app settings during the intervention. 
ENT Group



ET Group

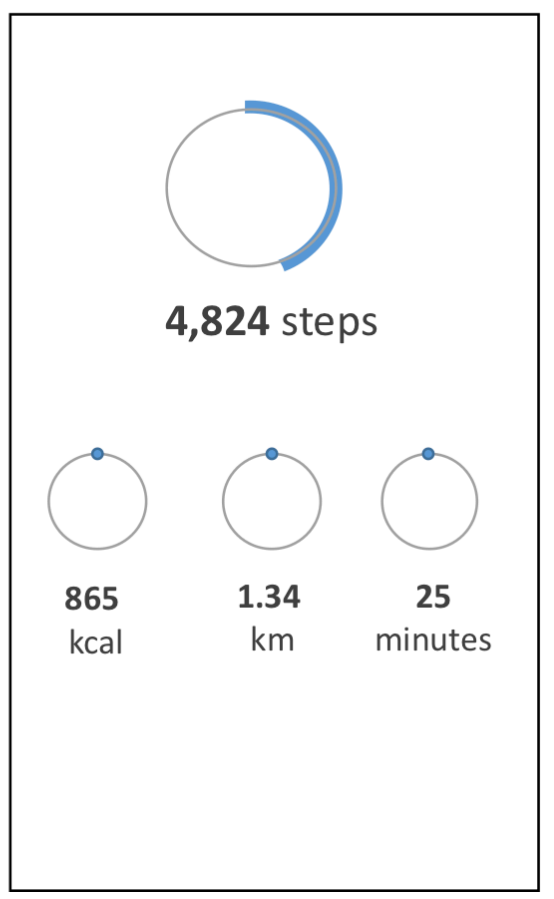

Figure 1. Visualisation of the fitness app interface.

\section{Blinding}

All participants were blinded to the study design and to the implementation of different experimental and control groups and conditions. After the study was completed, all participants received information regarding the study's research questions and design. During the generation of a sequence allocation, the researchers were blinded to the participants' identity. During the pre- and post-tests and the intervention, the researchers were in direct contact with the participants, and thus not blinded to the participants' identity or allocation. During the data analysis, all data was managed on the basis of the participants' code. Thus again, the researchers were blinded to the participants' identity, but not to the participants' group allocation. 


\section{Measures}

\section{Physical activity}

Physical activity was assessed via a German version of the Godin Leisure Time for Exercise Questionnaire (LTEQ; Shephard, 1997). The participants were asked to estimate the amount of strenuous, moderate, and light physical activity in units of 15 minutes referring to the past week. In the data analysis, a total score of physical activity was calculated, using the formula provided by Shepard (1997): total physical activity $=(9 \mathrm{x}$ number of units of strenuous exercise $)+(5 \mathrm{x}$ number of units of moderate exercise $)+(3 \mathrm{x}$ number of units of light exercise). For additional detailed analysis, the single scores of strenuous, moderate, and light exercise units were used. In the screening online questionnaire, physical activity was assessed via a short form single item, i.e., "for how many hours have you engaged in exercise during the past week?" in hours per week.

\section{Basic needs satisfaction}

To assess exercise basic needs satisfaction, the German psychological needs satisfaction in exercise scale (Rackow et al., 2013) was used. The instrument entails eleven items. The three scales autonomy (3 items; e.g., "I feel I have the opportunity to make choices with respect to the way I exercise"), competence (4 items; e.g., "I feel I execute the exercises of my training programme very effectively"), and relatedness (4 items; e.g., "I feel connected to the people I interact with while we exercise") were assessed on a 7-point Likert scale $(1=$ not agree; $7=$

fully agree). Reliability and validity have been shown to be satisfactory (Rackow et al., 2013).

\section{Perceived autonomy support}

To assess the perceived autonomy support provided by the fitness apps, a German version of the Health Care Climate Questionnaire (HCCQ; Schmidt et al., 2012) was used. A short form of three items was used: "I feel that the fitness app has provided me choices and options"; 
"The fitness app conveys confidence in my ability to make changes"; "I feel a lot of trust in the fitness tracker function of the fitness app". The items were assessed on a 7-point Likert scale $(1=$ not agree $7=$ fully agree $)$. Reliability and validity have been shown to be satisfactory (Schmidt et al., 2012). As the scale is referring to the autonomy support provided by the fitness app during the intervention, these items were assessed in the post-test in the ET and ENT groups.

\section{Motivation regulation}

In this study, the German instrument for measuring the self-concordance of sport- and exercise-related goals (SKK-scale; Seelig \& Fuchs, 2006) was applied. The SKK-scale consists of twelve items, measuring the scales intrinsic, identified, introjected, and external regulations (each 3 items) on a 6-point Likert scale $(1=$ not agree; $6=$ fully agree $)$. Validity tests indicated the instrument to be robust (Seelig \& Fuchs, 2006).

\section{Statistical Methods}

The data was analysed via the interface $\mathrm{R}$ statistics (R Core Team, 2016). In preliminary analyses, means and standard deviations of all scales (i.e., overall basic needs satisfaction, autonomy, competence and relatedness need satisfaction and total physical activity including the subscales of strenuous, moderate, and light exercise) were calculated for pre-test and posttest. Also, descriptive statistics of demographical variables (i.e., age, gender, educational status, student status) were calculated. The reliability of each scale was estimated via Cronbach's $\alpha$ (Cronbach, 1951) for both pre-test and post-test assessment. Potential group differences in baseline data were analysed via $\chi^{2}$-tests for categorical data and via analyses of variance (ANOVAs) for interval scaled data. If significant mean group differences were found, post-hoc pairwise t-tests were conducted. If group differences in demographical data existed, it was of interest whether these differences could have affected the results of the main analyses. Thus, in this case, complimentary analyses were conducted, repeating all main 
analyses and entering the referring demographical variables as covariates in analyses of variance (ANCOVAs).

\section{Main analysis}

In the main analysis, mean group differences between the ET, ENT, and control groups were calculated for autonomy, competence, and relatedness need satisfaction, for intrinsic, identified, introjected, and extrinsic motivation regulation, for amotivation, for total physical activity, and the subscales of strenuous, moderate, and light exercise. Therefore, repeated measure ANOVAs were conducted, using a Bonferroni correction. If overall mean differences were indicated in the analyses, post-hoc t-tests with pairwise group comparisons were conducted.

\section{Results}

In total, $N=152$ participants meeting the inclusion criteria were allocated to the groups and all participated in the pre-test. Each $n=50$ participants were recruited for the ET and ENT groups. Although it was planned to recruit 50 participants per group, $n=52$ participants were allocated to the control group due to simultaneous participation in the screening questionnaire (Figure 2). In total, data of $n=48$ participants in the ET group, $n=47$ participants in the ENT group, and $n=47$ participants in the control group was included in the analysis. The intervention time started on $3^{\text {rd }}$ January 2018 and ended after the pre-defined intervention time on $5^{\text {th }}$ June 2018.

In the screening questionnaire, participants reported to exercise $M=2.29(S D=1.19)$ hours per week (see Table 1). Here, no significant group differences were found. Also, no group differences in height and weight were found. Overall, mean age was $M=24.43(S D=4.61)$, and significant group differences were found $(F[2,139]=10.300, p<.001)$. Specifically, participants in the control group $(M=22.09, S D=3.96)$ were significantly younger (both $p<$ $.001)$ than participants in the ET $(M=25.67, S D=4.48)$ and $\mathrm{ENT}(M=25.51, S D=4.75)$ 
groups. However, no age differences between ET and ENT groups were found. The overall percentage of female was $20.42 \%$ and significant group differences were found $\left(\chi^{2}[2]=\right.$ $1.980, p=.372$ ). Specifically, $72.9 \%$ of the ET group, and each $83.0 \%$ of ENT and control groups were female. With regards to the measures screened in the fully randomized ET and ENT groups, no significant differences were observed in any of the variables assessed in the screening questionnaire.

\section{Pre-test}

Descriptive statistics of the data assessed in the pre- and post-tests are provided in Table 1. With regards to physical activity, it has been recommended to cover at least ten units of moderate exercise or five units of strenuous exercise per week (WHO, 2010). According to the LTEQ measures assessed in the pre-test, this standard was reached by $61.27 \%$ (pre-test) and $64.79 \%$ (post-test) of the participants in this study.

With regards to motivation regulation, no group differences were observed for all scales of motivation regulation (i.e., autonomous motivation, controlled motivation, and amotivation). In the analysis of basic needs satisfaction, significant group differences were observed in the pre-test for autonomy scale $(F[2,139]=3.960, p=.021)$, and relatedness scale $(F[2,139]=4.014, p=.020)$. Post-hoc-tests revealed group differences with regards to autonomy scale between ET and ENT groups $(p=.039)$ and ENT and control groups $(p=$ $.045)$, and with regards to relatedness scale between ET and ENT groups $(p=.022)$. Although the assessment of physical activity in the screening questionnaire (hours per week) revealed no group differences, mean differences in LTEQ scores were observed in the post-test $(F[2$, $139]=3.655, p=.028)$. Post-hoc-tests revealed mean differences between ENT and control groups $(p=.025)$. Cronbach's alpha levels for all subscales - except for extrinsic motivation in the pre-test and autonomy need satisfaction at both measurements - exceeded .70, meeting acceptable criteria (Table 1). 


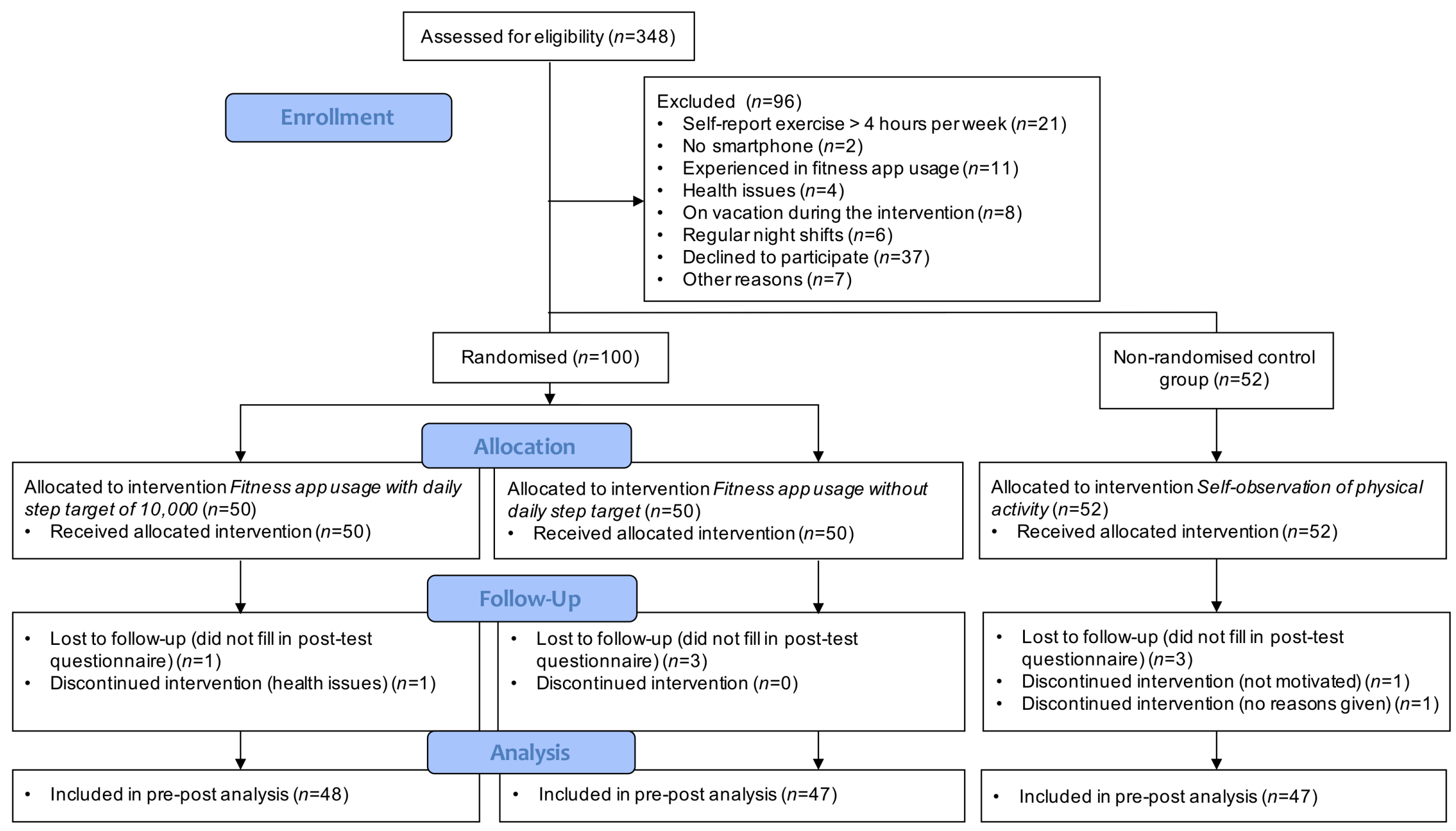

Figure 2. CONSORT Flow-Chart of this study. 
Table 1. Descriptive Statistics for Basic Needs, Motivation, and Physical Activity measured at Pre- and Post-Tests.

\begin{tabular}{|c|c|c|c|c|c|c|c|c|c|c|c|c|c|c|}
\hline \multirow[b]{3}{*}{ Variable } & \multicolumn{7}{|c|}{ Pre-Test } & \multicolumn{7}{|c|}{ Post-Test } \\
\hline & \multicolumn{2}{|c|}{ ET } & \multicolumn{2}{|c|}{ ENT } & \multicolumn{2}{|c|}{ Control } & \multirow[b]{2}{*}{$\alpha$} & \multicolumn{2}{|c|}{ ET } & \multicolumn{2}{|c|}{ ENT } & \multicolumn{2}{|c|}{ Control } & \multirow[b]{2}{*}{$\alpha$} \\
\hline & $M$ & $(S D)$ & $M$ & $(S D)$ & $M$ & $(S D)$ & & $M$ & $(S D)$ & $M$ & $(S D)$ & $M$ & $(S D)$ & \\
\hline \multicolumn{15}{|l|}{ Basic Needs } \\
\hline Autonomy & 5.31 & $(0.94)$ & 4.84 & $(0.98)$ & 5.30 & $(0.91)$ & .56 & 5.59 & $(0.99)$ & 5.00 & $(1.01)$ & 5.49 & $(0.88)$ & .59 \\
\hline Competence & 4.95 & $(1.10)$ & 4.83 & $(1.02)$ & 5.17 & $(0.95)$ & .82 & 5.07 & $(0.96)$ & 4.96 & $(1.05)$ & 5.31 & $(0.84)$ & .76 \\
\hline Relatedness & 5.29 & $(1.00)$ & 4.65 & $(1.34)$ & 5.16 & $(1.15)$ & .86 & 5.28 & $(0.99)$ & 4.61 & $(1.23)$ & 5.15 & $(1.25)$ & .87 \\
\hline \multicolumn{15}{|l|}{ Motivation } \\
\hline Intrinsic & 4.65 & (1.11) & 4.33 & $(1.21)$ & 4.58 & $(1.06)$ & .81 & 4.55 & $(1.04)$ & 4.29 & $(1.03)$ & 4.64 & $(1.02)$ & .79 \\
\hline Identified & 5.18 & $(0.66)$ & 5.19 & $(0.62)$ & 5.38 & $(0.53)$ & .70 & 4.91 & $(0.73)$ & 5.01 & $(0.92)$ & 5.10 & $(0.66)$ & .73 \\
\hline Introjected & 3.62 & $(0.97)$ & 3.67 & (1.04) & 3.79 & $(1.08)$ & .81 & 3.41 & $(1.16)$ & 3.67 & $(1.22)$ & 3.23 & $(1.31)$ & .77 \\
\hline Extrinsic & 1.65 & $(0.77)$ & 1.60 & $(0.71)$ & 1.55 & $(0.62)$ & .61 & 1.64 & $(0.77)$ & 1.74 & $(0.88)$ & 1.45 & $(0.75)$ & .76 \\
\hline Amotivation & 1.24 & $(0.42)$ & 1.40 & $(0.80)$ & 1.31 & $(0.45)$ & .90 & 1.39 & $(0.60)$ & 1.56 & $(0.84)$ & 1.30 & $(0.51)$ & .81 \\
\hline Physical Activity & 16.71 & $(10.01)$ & 13.14 & $(7.91)$ & 18.34 & $(10.49)$ & & 26.10 & $(21.51)$ & 22.87 & $(17.73)$ & 22.79 & $(19.21)$ & \\
\hline Strenuous & 5.52 & $(4.21)$ & 4.30 & $(3.32)$ & 5.74 & $(4.19)$ & & 8.10 & (11.99) & 5.13 & $(5.51)$ & 6.62 & $(8.59)$ & \\
\hline Moderate & 8.35 & $(6.78)$ & 6.36 & $(4.24)$ & 9.49 & $(8.11)$ & & 9.98 & $(9.47)$ & 10.06 & $(8.54)$ & 8.00 & $(6.48)$ & \\
\hline Light & 2.83 & $(3.77)$ & 2.48 & $(3.60)$ & 3.11 & $(4.15)$ & & 9.02 & (13.45) & 7.68 & $(8.45)$ & 8.17 & $(12.11)$ & \\
\hline
\end{tabular}

Note. ET, experimental target group; ENT, experimental no target group. 


\section{Post-test}

In the post-tests, significant group differences were observed with regards to autonomy $(F(2$, $139)=5.124, p=.007)$ and relatedness need satisfaction $(F[2,139]=4.434, p=.014)$. Posthoc-tests revealed that with regards to autonomy, significant differences between ET and ENT groups $(p=.009)$ and between ENT and control groups $(p=.038)$ were observed. For relatedness, significant differences between ET and ENT groups were observed $(p=.016)$.

\section{Main Analyses}

\section{Physical activity}

In the analyses of the LTEQ targeting physical activity, a significant time effect was found (see Table 2). Post-hoc-tests revealed significant changes in physical activity in the ET group $(t[47]=-2.827, p=.007)$ and in the ENT group $(t[46]=-4.291, p<.001)$, but not in the control group. No group or time-group interaction effects were found. In the analyses of the subscales of light, moderate, and strenuous exercise, no time, group, or time-group interaction effects were found for strenuous exercise. For moderate exercise, a time-group interaction effect was found. Post-hoc-tests revealed a significant increase of moderate physical activity in the ENT group $(t[46]=-3.065, p=.004)$, but not in the ET and control groups. With regards to light physical activity, a significant time effect was found. Post-hoc-tests revealed significant changes in the pre-post assessment for ET group $(t[47]=-3.242, p=.002)$, ENT group $(t[46]=-4.359, p<.001)$, and control group $(t[46]=-3.014, p=.004)$.

\section{Basic needs satisfaction}

With regards to autonomy, a significant time effect was found (Table 2). A post-hoc test revealed a significant increase in autonomy for the ENT group $(t[47]=-2.415, p=.020)$, but not for the ET and control groups. Also, a significant group effect was found. A post-hoc-test revealed significant differences between ET and ENT groups $(p<.001)$, and between ENT 
and control groups $(p=.002)$. However, no time-group-interaction effect was observed. With regards to competence, no significant group, time, or time-group interaction effects were found. With regards to relatedness, a significant group effect was found. A post-hoc-test revealed significant differences between ET and ENT groups $(p<.001)$ and between ENT and control groups $(p=.006)$. No significant time or time-group interaction effects were found. With regards to the perceived autonomy support tested in the post-test for the ET and ENT groups the post-test, no significant difference was found $(F[1,91]=0.169, p=.682)$.

\section{Motivation regulation}

With regards to intrinsic motivation, no time, group, or time-group interaction effects were found. For identified motivation, a significant time effect (Table 2) was found. Post-hoc-tests revealed a significant decrease in identified motivation for the ET group $(t[47]=2.232, p=$ $.030)$ and for the control group $(t[46]=2.954, p=.005)$, but not for the ENT group. No group effects or time-group interaction effects were found. With regards to introjected motivation, a significant time effect was found. Post-hoc-tests revealed a significant decrease in identified motivation for the control group $(t[46]=4.283, p<.001)$. Also, a significant time-group interaction effect was found. No group effect was found. For extrinsic motivation, no time, group, or time-group interaction effects were found. With regards to amotivation, a significant time effect was found. Post-hoc-tests revealed a significant increase in amotivation for the ENT group $(t[46]=-2.038, p=.047)$, but not for the ET and control groups. No group or time-group interaction effects were found.

\section{Complimentary analyses}

As group differences referring to age and education were found in the preliminary analyses, all analyses were conducted again, entering age, education status, and student status as covariates. Here, in none of the results significantly differences compared to the results of the main analyses were found. 
Table 2. Results of the ANOVAs Targeting the Effects of Self-Tracking via Fitness Apps and Normalized Step Targets on Basic Needs Satisfaction, Motivation, and Physical Activity.

\begin{tabular}{|c|c|c|c|c|c|c|c|c|c|c|c|c|}
\hline \multirow[b]{2}{*}{ Variable } & \multicolumn{4}{|c|}{ Time } & \multicolumn{4}{|c|}{ Group } & \multicolumn{4}{|c|}{ Time x Group } \\
\hline & $d f$ & $F$ & $p$ & $\eta^{2}$ & $d f$ & $F$ & $p$ & $\eta^{2}$ & $d f$ & $F$ & $p$ & $\eta^{2}$ \\
\hline \multicolumn{13}{|l|}{$\underline{\text { Basic Needs }}$} \\
\hline Autonomy & 1,139 & 7.293 & $.008 * *$ & 0.012 & 2,139 & 5.884 & $.004 * *$ & 0.061 & 2,139 & 0.210 & .811 & $<0.001$ \\
\hline Competence & 1,139 & 2.931 & .089 & 0.004 & 2,139 & 2.9308 & .089 & 0.021 & 2,139 & 0.005 & .995 & $<0.001$ \\
\hline Relatedness & 1,139 & 0.104 & .747 & 0.001 & 2,139 & 4.984 & $<.001 * * *$ & 0.057 & 2,139 & 0.014 & .987 & $<0.001$ \\
\hline \multicolumn{13}{|l|}{$\underline{\text { Motivation }}$} \\
\hline Intrinsic & 1,139 & 0.235 & .622 & $<0.001$ & 2,139 & 1.318 & .271 & 0.017 & 2,139 & 0.670 & .513 & $<0.001$ \\
\hline Identified & 1,139 & 15.495 & $<.001 * * *$ & 0.029 & 2,139 & 1.348 & .263 & 0.014 & 2,139 & 0.262 & .770 & 0.001 \\
\hline Introjected & 1,139 & 9.352 & $<.001 * * *$ & 0.012 & 2,139 & 0.361 & .697 & 0.004 & 2,139 & 3.751 & $<.001 *$ & 0.010 \\
\hline Extrinsic & 1,139 & 0.031 & .863 & $<0.001$ & 2,139 & 0.878 & .418 & 0.010 & 2,139 & 1.680 & .190 & 0.004 \\
\hline Amotivation & 1,139 & 4.951 & $.027 *$ & 0.006 & 2,139 & 1.454 & .237 & 0.017 & 2,139 & 1.538 & .219 & 0.003 \\
\hline Physical Activity & 1,139 & 24.274 & $<.001 * * *$ & 0.062 & 2,139 & 1.013 & .366 & 0.009 & 2,139 & 1.143 & .322 & 0.006 \\
\hline Strenuous & 1,139 & 3.737 & .055 & 0.010 & 2,139 & 1.839 & .163 & 0.016 & 2,139 & 0.615 & .542 & 0.003 \\
\hline Moderate & 1,139 & 1.453 & .231 & 0.004 & 2,139 & 0.114 & .892 & 0.001 & 2,139 & 3.664 & $.028 *$ & 0.020 \\
\hline Light & 1,139 & 34.119 & $<.001 * * *$ & 0.094 & 2,139 & 0.206 & .814 & 0.002 & 2,139 & 0.143 & .867 & $<0.001$ \\
\hline
\end{tabular}

Note. ${ }^{*} p<.05, * * p<.01, * * * p<.001 ;$ bold, $p<.05$ 


\section{Discussion}

Using the well-established SDT as a theoretical framework, it was the aim of this study to investigate the effects of healthcare technology, specifically a fitness app and wearable, and the implementation of a normalized health target on basic needs satisfaction, motivation and health behaviour (i.e., physical activity). It was hypothesized that the implementation of a fitness app and wearable to support physical activity tracking has a positive effect on physical activity. Also, it was hypothesized that the implementation of a normalized step target (i.e., 10,000 steps per day) would result in decreased levels of autonomy need satisfaction, autonomous motivation, and in increased levels of controlled motivation and amotivation compared to no implementation of such targets.

In this study, total physical activity levels significantly increased in participants in both experimental groups that were provided with a fitness wearable and app to track their physical activity. This effect was not observed in the control group with participants tracking their physical activity without technology support. The results indicate that the implementation of health technology such as fitness apps and wearables to track physical activity can have beneficial effects and can be an important means to support daily physical activity. Thus, Hypothesis 1 could be confirmed. Across studies, it has been indicated that the implementation of physical activity trackers, and specifically fitness apps can foster higher activity levels, such as step counts (e.g., Donnachie et al., 2017; Glynn et al., 2014; Kerner \& Goodyear, 2017). Furthermore, increases in moderate physical activity were only observed in the ENT group. Similarly, in a study reviewing the effects of specific Fitbit technology on step count, it was concluded that the majority of Fitbit-based interventions had a positive effects on moderate to vigorous physical activity, but not on the number of steps (Choi et al., 2016; Mishra et al., 2017). Furthermore, the support of tracking devices have been found to be effective for precise decision making during the training process, and can assist to improve 
training outcomes (Malone et al., 2017). Also, the implementation of objective technologybased feedback can lead to autonomy need satisfaction, habit formation, internalisation of motivation, and thus, to maintenance of physical activity (Donnachie et al., 2017; Stawarz et al., 2015). However, mixed results of fitness tracker usage on physical activity and specifically step counts have been reviewed and meta-analysed (Romeo et al., 2019; Schoeppe et al., 2016). In this context, it has been indicated that the effects might depend of motivational determinants. These motivational determinants can vary across app setting, specifically providing autonomy supportive vs. controlling conditions. For example, one such controlling condition can be created via the implementation of normalized step targets (Goodyear et al., 2017; Kerner et al., 2019).

In previous studies investigating the effect of specific step targets in fitness app users (e.g., 10,000 steps per day), and specifically in Fitbit users on motivation, decreases in autonomy need satisfaction were found (Kerner \& Goodyear, 2017, Kerner et al., 2019). In contrast, in this study no changes in autonomy need satisfaction were found in the ET group. Thus, Hypothesis 2 could not be confirmed. Interestingly, unlike in the ET and control groups, autonomy need satisfaction increased in participants allocated to the ENT group where no normalized step target was implemented. Consequently, it can be assumed that the technology mediated visibility of progress within the fitness app interface can leave room for own choices and autonomy.

With regards to motivation regulation, an increase in amotivation in the ENT group was found. As the ENT group was asked not to set own goals in the app settings, the complete lack of specific targets might have resulted in a state of disorientation and amotivation that can be described by not seeing the point in exercising. However, it should be noted that the increase of amotivation found in the ENT group was not associated with a lack of physical activity - in contrast, the ENT groups reported the highest increase in physical activity, and 
especially moderate physical activity compared to the control group and also compared to the ET group. Furthermore, decreases in specific forms of motivation were found in the ET and control groups in this study. Specifically, decreases in identified motivation were found in the ET and the control groups, and a decrease in introjected motivation was found in the control group. In this context, it has been meta-analysed that autonomous forms of motivation (i.e., internal and identified motivation) can lead to enhanced physical health, such as physical activity, and mental health (Ng et al., 2012). Also, it has been meta-analysed that controlled forms of motivation (i.e., introjected and external motivation) can be negatively associated with mental health — and thus represent undesired health outcomes— but are also connected with higher levels of physical health, such as physical activity. Similarly, it has been discussed that the implementation of normalized step targets can lead to feelings of pressure and fear and has been found to decreased levels of autonomous motivation, and even postinterventional decreases in physical activity (Kerner et al., 2019; Kerner \& Goodyear, 2017; Lupton, 2014).

Thus, corresponding with the empirically supported motivational sequence postulated in the SDT health model (Ng et al., 2012; Ryan \& Deci, 2008; Teixeira et al., 2012), autonomy need satisfaction increased in the ENT group, which was consequently associated with the most pronounced increase in physical activity. In the ET and control groups, no increases in autonomy need satisfaction were found, and thus, in both groups, autonomous forms of motivation decreased. Additionally, controlled forms of motivation increased in the control group, which was associated with a lower increase in physical activity.

\section{Strengths and Limitations}

This study was a six-week RCT that was conducted under controlled conditions (i.e., random sampling, pre- and post-tests under lab conditions, with participants all using identical fitness tracker devices and apps in a sample of 152 participants). Also, high adherence rates were 
reached in this study. In addition to the two experimental groups, an additional control group was implemented to assess the effect of technology mediated self-tracking compared no nontechnology mediated self-tracking. The control group was separately acquired in the same areas, via the same media, and via an identically looking flyer that was blind to the intervention (i.e., the implementation of fitness tracker and wearable usage). In another option, all three groups could have been recruited via the same flyer which would have included the obligatory information that the participants might (or might not) receive a fitness tracker. This was important for ethical reasons as fitness app usage includes the disclosure of personal data. In this scenario, however, it was expected that the group not being provided with a fitness tracker could have perceived inferiority to the participants who were provided with fitness tracker groups, potentially leading to undesired and hardly controllable group effects. Therefore, we decided against this option. In this study, group differences in both age and gender were found that not only existed between the not fully randomized control group and experimental groups but also between the two fully randomized ET and ENT groups. Therefore, to control for potential effects, we conducted additional analyses entering these variables as covariates. However, no changes in effects were found in the referring analyses. These findings are in line with recent reviews indicating that the effects of fitness app usage are robust to age differences (Romeo et al., 2019).

In this study, we investigated prominent and widely used constructs that were based on SDT. Correspondingly, we used well-established assessment tools that have been demonstrated good reliability and validity across diverse studies (e.g., Rackow et al., 2013; Seelig \& Fuchs, 2006; Shephard, 1997). However, the reliability coefficients of the extrinsic motivation in the pre-test and autonomy scale at pre- and post-tests did not exceed acceptable levels of .70. Thus, the referring results should be interpreted with care. 
In addition to the assessment of basic needs satisfaction, motivation regulation, and physical activity, we were interested in whether the lack of normalized goals would lead to higher levels of perceived autonomy support compared to the implementation of such a goal. Here, no group differences between ET and ENT groups were observed. These results can be explained by the fact that no specific guidance for autonomous goal setting was provided in the ENT group. In this context, it could have been interesting to assess whether the implementation of normalized step targets vs. the lack of a step target would have induced higher levels of perceived autonomy thwarting in the ET group compared to the ENT group. This point is an interesting question to consider in future studies.

\section{Practical Implications and Conclusion}

It was the aim of this study to examine the effects of self-tracking via fitness apps and the implementation of a normalized step target on basic needs satisfaction, motivation, and physical activity by using SDT as a theoretical basis. Thus, first, it was found that the implementation of technology support to track and visualize can foster autonomy need satisfaction, prevent from a decrease in motivation, and can foster increases in physical activity levels. Therefore, practitioners in healthcare have new options to implement and form routines in physical activity behaviour. This point might be especially relevant for groups that can highly benefit from support in the establishment of activity behaviour, such as patients suffering from type II-diabetes or from depressive disorders (Chimen et al., 2012; Ströhle, 2009). For example, it has been found that a Fitbit based intervention program in depressed alcohol-depended women led to increased physical activity levels and reductions in depressive and anxiety symptoms (Abrantes et al., 2017).

Second, the implementation of normalized step targets (e.g., 10,000 steps per day) was associated with lower increase in physical activity, a lack of autonomy need satisfaction and decreased levels of identified motivation. Also, the results of this study indicate that a 
complete absence of targets can lead to a state of amotivation or a lack of orientation.

Correspondingly, it has been reviewed that fitness app interventions (e.g., targeting physical activity) that are based on behaviour change theories such as goal setting, are more effective than interventions that lack such foundation, and it has been recommended to implement behavioural plans (Bakker et al., 2016; Schoeppe et al., 2016). Thus, it can be highly promising to investigate the additional effect of individualized goal setting on autonomy need satisfaction, autonomous motivation, and physical activity. Moreover, these interventions should be designed in an autonomy supportive way, e.g., by allowing the user to experience own choice and to set goals that can lead to the desired outcomes. In this context, SMART goal setting (i.e., Specific, Measurable, Attractive, Realistic, and Terminated goals) has been found to be highly effective, for example on motivated behavioural outcomes and positive affect (Johnson et al., 2014; McCarthy et al., 2010). Therefore, practitioners implementing such techniques might highly benefit from combining individual support in goal setting with the implementation of a technology to track physical activity. With regards to the advised length of interventions, two points should be considered. First, increasing dropout rates in fitness app usage have been observed as reviewed by Schoeppe et al. (2016), the participants' average usage of fitness app programs (e.g., the Australian 10,000 steps app and website) is five to six weeks, indicating a rapid decline in usage. Still, a combined usage of media (e.g., website and app) was associated with longer duration of usage, i.e., about eight weeks. The rapid dropout is assumed to be a consequence of lacking commitment and rather transient, irregular usage of fitness apps (Dennison et al., 2013). Second, short term interventions $(<3$ months) have been found to be more effective that long-term fitness app-based interventions (Romeo et al., 2019 for review). Thus, it can be helpful to implement an intervention time that can assist in shaping a behaviour routine and can support the user to establish a shift from behaviour that is first guided by controlled motivation, but then can shift to autonomous 
motivation, making the support of technical devices less relevant after time (Donnachie et al., 2017). To support this progress, fitness app-based interventions should be designed with autonomy-supportive tools and functions and might even benefit from additional coaching in effective goal setting. Therefore, future studies are needed that investigate the effects of autonomous goal setting, the implementation of coaching how to practice effective goal setting in studies under controlled conditions. Furthermore, the examination of such effects can be especially interesting in special user groups, for example in patients with physical injuries, during rehabilitation, or during the treatment of depressive disorders.

\section{Declarations}

We have no conflict of interest to declare. 


\section{References}

Abrantes, A. M., Blevins, C. E., Battle, C. L., Read, J. P., Gordon, A. L., \& Stein, M. D. (2017). Developing a Fitbit-supported lifestyle physical activity intervention for depressed alcohol dependent women. Journal of Substance Abuse Treatment, 80, 8897.

Bakker, D., Kazantzis, N., Rickwood, D., \& Rickard, N. (2016). Mental health smartphone apps: Review and evidence-based recommendations for future developments. JMIR Mental Health, 3(1), e7.

Bouchard, C., Blair, S. N., \& Haskell, W. L. (2018). Physical activity and health. Human Kinetics.

Busch, L. (2019). The Psychology of Technology-An Interdisciplinary Approach to Understand the Role of Trust in Fitness App Usage. University of Münster.

Chimen, M., Kennedy, A., Nirantharakumar, K., Pang, T. T., Andrews, R., \& Narendran, P. (2012). What are the health benefits of physical activity in type 1 diabetes mellitus? A literature review. Diabetologia, 55(3), 542-551.

Choi, J., Hyeon Lee, J., Vittinghoff, E., \& Fukuoka, Y. (2016). mHealth physical activity intervention: A randomized pilot study in physically inactive pregnant women. Maternal and Child Health Journal, 20(5), 1091-1101.

Cronbach, L. J. (1951). Coefficient alpha and the internal structure of tests. Psychometrika, 16(3), 297-334.

Deci, E. R., \& Ryan, S. C. (1985). Intrinsic motivation and self-determination in human behaviour. Plenum, New York.

Dennison, L., Morrison, L., Conway, G., \& Yardley, L. (2013). Opportunities and challenges for smartphone applications in supporting health behavior change: Qualitative study. Journal of Medical Internet Research, 15(4), e86. https://doi.org/10.2196/jmir.2583

Donnachie, C., Wyke, S., Mutrie, N., \& Hunt, K. (2017). 'It's like a personal motivator that you carried around wi'you': Utilising self-determination theory to understand men's experiences of using pedometers to increase physical activity in a weight management programme. International Journal of Behavioral Nutrition and Physical Activity, 14(1), 61 .

DRKS. (2019). https://www.drks.de/drks_web/

Field, A. E., Coakley, E. H., Must, A., Spadano, J. L., Laird, N., Dietz, W. H., Rimm, E., \& Colditz, G. A. (2001). Impact of overweight on the risk of developing common 
chronic diseases during a 10-year period. Archives of Internal Medicine, 161(13), $1581-1586$.

Gagné, M., \& Deci, E. L. (2005). Self-determination theory and work motivation. Journal of Organizational Behavior, 26(4), 331-362.

Global Studies-Fitness tracking. http://www.gfk.com/global-studies/global-studies-fitnesstracking/

Glynn, L. G., Hayes, P. S., Casey, M., Glynn, F., Alvarez-Iglesias, A., Newell, J., Ólaighin, G., Heaney, D., O’Donnell, M., \& Murphy, A. W. (2014). Effectiveness of a smartphone application to promote physical activity in primary care: The SMART MOVE randomised controlled trial. Br J Gen Pract, 64(624), e384-e391.

Goodyear, V. A., Kerner, C., \& Quennerstedt, M. (2017). Young people's uses of wearable healthy lifestyle technologies; surveillance, self-surveillance and resistance. Sport, Education and Society, 1-14.

Helmert, U., \& Strube, H. (2004). Die Entwicklung der Adipositas in Deutschland im Zeitraum von 1985 bis 2002. Das Gesundheitswesen, 66(07), 409-415. https://doi.org/10.1055/s-2004-813324

Higgins, J. P. (2016). Smartphone Applications for Patients' Health and Fitness. The American Journal of Medicine, 129(1), 11-19. https://doi.org/10.1016/J.AMJMED.2015.05.038

Johnson, C., Moore, E., \& Thornton, M. (2014). A SMART Approach to Motivating Students in Secondary Physical Education: Editor: Ferman Konukman. Journal of Physical Education, Recreation and Dance, 85(4), 42-44.

Kerner, C., Burrows, A., \& McGrane, B. (2019). Health wearables in adolescents: Implications for body satisfaction, motivation and physical activity. International Journal of Health Promotion and Education, 57(4), 191-202.

Kerner, C., \& Goodyear, V. A. (2017). The Motivational Impact of Wearable Healthy Lifestyle Technologies: A Self-determination Perspective on Fitbits With Adolescents. American Journal of Health Education, 1-11.

Lupton, D. (2014). Apps as artefacts: Towards a critical perspective on mobile health and medical apps. Societies, 4(4), 606-622.

Maher, C., Ferguson, M., Vandelanotte, C., Plotnikoff, R., De Bourdeaudhuij, I., Thomas, S., Nelson-Field, K., \& Olds, T. (2015). A Web-Based, Social Networking Physical Activity Intervention for Insufficiently Active Adults Delivered via Facebook App: 
Randomized Controlled Trial. Journal of Medical Internet Research, 17(7), e174. https://doi.org/10.2196/jmir.4086

Malone, J. J., Lovell, R., Varley, M. C., \& Coutts, A. J. (2017). Unpacking the black box: Applications and considerations for using GPS devices in sport. International Journal of Sports Physiology and Performance, 12(Suppl 2), S2-18.

McCarthy, P. J., Jones, M. V., Harwood, C. G., \& Davenport, L. (2010). Using goal setting to enhance positive affect among junior multievent athletes. Journal of Clinical Sport Psychology, 4(1), 53-68.

Middelweerd, A., Mollee, J. S., van der Wal, C. N., Brug, J., \& te Velde, S. J. (2014). Apps to promote physical activity among adults: A review and content analysis. International Journal of Behavioral Nutrition and Physical Activity, 11(1), 97. https://doi.org/10.1186/s12966-014-0097-9

Mishra, A., Nieto, A., \& Kitsiou, S. (2017). Systematic review of mHealth interventions involving Fitbit activity tracking devices. 2017 IEEE International Conference on Healthcare Informatics (ICHI), 455-455.

Ng, J. Y., Ntoumanis, N., Thøgersen-Ntoumani, C., Deci, E. L., Ryan, R. M., Duda, J. L., \& Williams, G. C. (2012). Self-determination theory applied to health contexts: A metaanalysis. Perspectives on Psychological Science, 7(4), 325-340.

Niemiec, C. P., \& Ryan, R. M. (2009). Autonomy, competence, and relatedness in the classroom: Applying self-determination theory to educational practice. School Field, $7(2), 133-144$.

Ntoumanis, N. (2001). A self-determination approach to the understanding of motivation in physical education. British Journal of Educational Psychology, 71(2), 225-242.

Poushter, J. (2016). Smartphone ownership and internet usage continues to climb in emerging economies. Pew Research Center, 22, 1-44.

Prugger, C., \& Keil, U. (2007). Entwicklung der Adipositas in Deutschland-Größenordnung, Determinanten und Perspektiven. DMW-Deutsche Medizinische Wochenschrift, 132(16), 892-897.

Questback GmbH. (2018). Unipark. www.unipark.de

R Core Team. (2016). R: A language and environment for statistical computing. $\mathrm{R}$ Foundation for Statistical Computing. https:/www.R-project.org/

Rackow, P., Scholz, U., \& Hornung, R. (2013). The German Psychological Need Satisfaction in Exercise Scale. Swiss Journal of Psychology, 72(3), 137-148. https://doi.org/10.1024/1421-0185/a000107 
Riley, C., Lee, M., Cooper, Z., Fairburn, C. G., \& Shafran, R. (2007). A randomised controlled trial of cognitive-behaviour therapy for clinical perfectionism: A preliminary study. Behaviour Research and Therapy, 45(9), 2221-2231. Scopus. https://doi.org/10.1016/j.brat.2006.12.003

Romeo, A., Edney, S., Plotnikoff, R., Curtis, R., Ryan, J., Sanders, I., Crozier, A., \& Maher, C. (2019). Can smartphone apps increase physical activity? Systematic review and meta-analysis. Journal of Medical Internet Research, 21(3), e12053.

Ryan, R., \& Deci, E. (2017). Self-determination theory: Basic psychological needs in motivation, development, and wellness. Guilford Publications.

Ryan, R. M., \& Deci, E. L. (2008). A self-determination theory approach to psychotherapy: The motivational basis for effective change. Canadian Psychology/Psychologie Canadienne, 49(3), 186.

Ryan, R. M., Patrick, H., Deci, E. L., \& Williams, G. C. (2008). Facilitating health behaviour change and its maintenance: Interventions based on self-determination theory. The European Health Psychologist, 10(1), 2-5.

Schoeppe, S., Alley, S., Van Lippevelde, W., Bray, N. A., Williams, S. L., Duncan, M. J., \& Vandelanotte, C. (2016). Efficacy of interventions that use apps to improve diet, physical activity and sedentary behaviour: A systematic review. International Journal of Behavioral Nutrition and Physical Activity, 13(1), 127. https://doi.org/10.1186/s12966-016-0454-y

Schulz, K. F., Altman, D. G., \& Moher, D. (2010). CONSORT 2010 statement: Updated guidelines for reporting parallel group randomised trials. BMC Medicine, $8(1), 18$.

Seelig, H., \& Fuchs, R. (2006). Messung der sport- und bewegungsbezogenen Selbstkonkordanz. Zeitschrift Für Sportpsychologie, 13(4), 121-139. https://doi.org/10.1026/1612-5010.13.4.121

Shephard, R. (1997). Godin leisure-time exercise questionnaire. Med Sci Sports Exerc, 29(suppl 6), S36-S38.

Silva, M. N., Vieira, P. N., Coutinho, S. R., Minderico, C. S., Matos, M. G., Sardinha, L. B., \& Teixeira, P. J. (2010). Using self-determination theory to promote physical activity and weight control: A randomized controlled trial in women. Journal of Behavioral Medicine, 33(2), 110-122.

Soper, D. (2012). A-priori sample size calculator for multiple regression. http://www. Danielsoper. Com/Statcalc3/Calc. Aspx. 
Statista. (2017). U.S. wearable user penetration 2017, by age. Statista.

https://www.statista.com/statistics/739398/us-wearable-penetration-by-age/

Statista. (2018). Mobile Internet \& Apps. Statista.

https://www.statista.com/statistics/267346/average-apple-app-store-price-app/

Stawarz, K., Cox, A. L., \& Blandford, A. (2015). Beyond Self-Tracking and Reminders.

Proceedings of the 33rd Annual ACM Conference on Human Factors in Computing

Systems - CHI '15, 2653-2662. https://doi.org/10.1145/2702123.2702230

Ströhle, A. (2009). Physical activity, exercise, depression and anxiety disorders. Journal of Neural Transmission, 116(6), 777.

Teixeira, P. J., Carraça, E. V., Markland, D., Silva, M. N., \& Ryan, R. M. (2012). Exercise, physical activity, and self-determination theory: A systematic review. International Journal of Behavioral Nutrition and Physical Activity, 9(1), 78.

Tudor-Locke, C., Brashear, M. M., Johnson, W. D., \& Katzmarzyk, P. T. (2010).

Accelerometer profiles of physical activity and inactivity in normal weight, overweight, and obese U.S. men and women. International Journal of Behavioral Nutrition and Physical Activity, 7(1), 60. https://doi.org/10.1186/1479-5868-7-60

Walsh, J. C., Corbett, T., Hogan, M., Duggan, J., \& McNamara, A. (2016). An mHealth intervention using a smartphone app to increase walking behavior in young adults: A pilot study. JMIR MHealth and UHealth, 4(3).

Wang, J. B., Cadmus-Bertram, L. A., Natarajan, L., White, M. M., Madanat, H., Nichols, J. F., Ayala, G. X., \& Pierce, J. P. (2015). Wearable sensor/device (Fitbit One) and SMS text-messaging prompts to increase physical activity in overweight and obese adults: A randomized controlled trial. Telemedicine and E-Health, 21(10), 782-792.

Warburton, D. E., Charlesworth, S., Ivey, A., Nettlefold, L., \& Bredin, S. S. (2010). A systematic review of the evidence for Canada's Physical Activity Guidelines for Adults. International Journal of Behavioral Nutrition and Physical Activity, 7(1), 39.

West, J. H., Hall, P. C., Hanson, C. L., Barnes, M. D., Giraud-Carrier, C., \& Barrett, J. (2012). There's an app for that: Content analysis of paid health and fitness apps. Journal of Medical Internet Research, 14(3), e72. https://doi.org/10.2196/jmir.1977

WHO. (2010). Global Recommendations on Physical Activity for Health. https://apps.who.int/iris/bitstream/handle/10665/44399/9789241599979_eng.pdf?sequ en $\% 20$ ce $=1$

WHO. (2017). Overweight and obesity. http://www.who.int/gho/ncd/risk_factors/overweight/en/ 
Williams, D. M., Dunsiger, S., Emerson, J. A., Gwaltney, C. J., Monti, P. M., \& Miranda Jr, R. (2016). Self-paced exercise, affective response, and exercise adherence: A preliminary investigation using ecological momentary assessment. Journal of Sport and Exercise Psychology, 38(3), 282-291.

Zheng, Y.-L., Ding, X.-R., Poon, C. C. Y., Lo, B. P. L., Zhang, H., Zhou, X.-L., Yang, G.-Z., Zhao, N., \& Zhang, Y.-T. (2014). Unobtrusive sensing and wearable devices for health informatics. IEEE Transactions on Biomedical Engineering, 61(5), 1538-1554. 